IZA DP No. 4768

The Effect of Children on Specialization and Coordination of Partners' Activities

Anh T. Le

Paul W. Miller

February 2010 


\title{
The Effect of Children on Specialization and Coordination of Partners' Activities
}

\author{
Anh T. Le \\ Curtin University of Technology \\ and IZA \\ Paul W. Miller \\ Curtin University of Technology \\ and IZA
}

\section{Discussion Paper No. 4768 \\ February 2010}

\author{
IZA \\ P.O. Box 7240 \\ 53072 Bonn \\ Germany \\ Phone: +49-228-3894-0 \\ Fax: +49-228-3894-180 \\ E-mail: iza@iza.org
}

Any opinions expressed here are those of the author(s) and not those of IZA. Research published in this series may include views on policy, but the institute itself takes no institutional policy positions.

The Institute for the Study of Labor (IZA) in Bonn is a local and virtual international research center and a place of communication between science, politics and business. IZA is an independent nonprofit organization supported by Deutsche Post Foundation. The center is associated with the University of Bonn and offers a stimulating research environment through its international network, workshops and conferences, data service, project support, research visits and doctoral program. IZA engages in (i) original and internationally competitive research in all fields of labor economics, (ii) development of policy concepts, and (iii) dissemination of research results and concepts to the interested public.

IZA Discussion Papers often represent preliminary work and are circulated to encourage discussion. Citation of such a paper should account for its provisional character. A revised version may be available directly from the author. 
IZA Discussion Paper No. 4768

February 2010

\section{ABSTRACT \\ The Effect of Children on Specialization and Coordination of Partners' Activities ${ }^{\star}$}

This paper first documents the extent of the specialization in time use in couple families, and the impact of children on this specialization. It then examines the links between the time allocations of partners in couple families, the impact of children on these links, and the effects these factors have on specialization in time use. Children are shown to intensify the specialization in time use in couple families through reducing the apparent complementarity in time allocations of their parents.

JEL Classification: J13, J16, J22

Keywords: time allocations, gender, specialisation, coordination

Corresponding author:

Paul W. Miller

School of Economics and Finance

Curtin Business School

Curtin University of Technology

GPO Box U1987

Perth WA 6845

Australia

E-mail: Paul.Miller@curtin.edu.au

* The authors acknowledge financial assistance from the Australian Research Council. 


\section{THE EFFECT OF CHILDREN ON SPECIALIZATION AND COORDINATION OF PARTNERS' ACTIVITES}

\section{INTRODUCTION}

Over the past three decades, economists have increasingly analysed decision making on time use within a family context. Families have been modelled as an arrangement that facilitates specialization. This specialization is based on the relative labor market potential of partners, and is usually argued to lead to men allocating additional time to labor market activities at the expense of household duties, and women allocating additional time to household duties at the expense of labor market activities (see, for example, Becker, 1991). Attempts to develop the dynamics of decision making within the family have seen a game theoretic approach adopted (see, for example Manser and Brown, 1980). The reaction curves used in this approach suggest some degree of implicit or explicit coordination of the activities of partners.

Many of the facts on the specialization in labor market activities by males, and in household activities by females, in couple families are well known. The information added to this conventional wisdom in this paper concerns the coordination of partners' activities within couple families, the effect of this coordination on the degree of specialization, and the impact of children on this coordination and specialization.

The paper is structured as follows. Section II introduces the data set used. Section III first establishes the extent of specialization in couple families with and without children. It then documents the degree of coordination of partners' activities within these families and the interaction of coordination and specialization. Section IV provides a brief conclusion. 


\section{DATA}

The analyses presented in this paper are based on The Time Use Survey 2006 undertaken by the Australian Bureau of Statistics. This survey uses a Time Diary approach. Information was collected from 3,626 households. There were 3,793 families comprising 6,902 within-scope individuals aged 15 or more years. Most of these individuals provided time diaries for two days, giving a sample of 13,617 diary days. Detailed data were collected on time use, along with standard demographic information. The data are available in the form of a Confidentialized Unit Record File. $^{1}$

The broad categories of time use that can be examined with these data are: (a) personal care; (b) employment-related; (c) education; (d) domestic; (e) child care; (f) purchasing goods and services; (g) voluntary work and care; (h) social and community interaction; (i) recreation and leisure; and (i) other. ${ }^{2}$ However, for the current set of analysis, this level of detail is not required. Hence, domestic activities will be aggregated with purchasing goods and services to form a "home duties" category, and social and community interaction combined with recreation and leisure to form a "leisure" category. Education, voluntary work and care, and undescribed will be aggregated to form a residual "other time uses" category. Thus a five-way disaggregation (with child care added to the residual other category) is used for the

\footnotetext{
${ }^{1}$ The basic file released on CD-ROM by the Australian Bureau of Statistics is used in the analyses that follow.

${ }^{2}$ Greater detail on each of these broad categories is available. For example, the domestic category can be disaggregated into specific uses such as food and drink preparation and cleanup, laundry and clothes care, other housework, grounds and animal care, home maintenance, and travel associated with domestic activities. Generally economists have focussed on broad categories of activities in time use studies.
} 
study of couple families without children, and a six-way disaggregation that gives explicit mention to child care is used in the study of couple families with children.

\section{TIME ALLOCATIONS IN COUPLE FAMILIES}

\section{(a) Specialization in Time Use}

Figure 1 provides information on the allocations of time in couple families without children (top panel) and with children (bottom panel). Comparison of males (left-hand diagram) and females (right-hand diagram) for each family type shows the degree of specialisation. Comparisons across family types for males or females shows the impact of children on this specialisation. These data cover the activities of individuals aged 20-64 years during Monday to Friday inclusive.

It is readily apparent from these data that males in couple families without children typically specialize in employment-related activities and females typically specialize in home duties. Thus, in this family type, males allocate 29 percent of their time to employment-related activities and eight percent of their time to home duties. In contrast, females in couple families without children allocate 18 percent of their time to employment-related activities and 15 percent of their time to home duties. ${ }^{3}$

Children intensify this specialization. Hence, the average time allocation to employment-related activities by males in couple families increases by four percentage points in the presence of children, and the average time allocated to home duties falls by one percentage point. Among females in couple families, the time

${ }^{3}$ Comparison of these data for couple families without children with similar data for singles further establishes the extensive specialization, by males in employmentrelated activities and by females in home duties, that is associated with the transition from the singles Lifecourse Stage to the couples Lifecourse Stage. 
allocated to employment-related activities falls by 4 percentage points in the presence of children, while the time allocated to home duties increases by three percentage points. As well, males in couple families with children allocate three percent of their time to child care whereas females in this family type allocate 10 percent of their time to child care. Further examination using regression analysis shows that young children are associated with more intense division of labor within the household than are older children. ${ }^{4}$

The extent of this specialization can be summarized using an index of dissimilarity analogous to that developed by Duncan and Duncan (1955) for the study of occupational segregation. As applied to these time budget data, this is given as $T D=0.5 \sum_{i=1}^{n}\left|T_{i m}-T_{i j}\right|$, where $T D$ is the overall time discrepancy between males and females, $T_{i j}$ is the time allocated to the $i^{\text {th }}$ activity by group $j, j=m$ for males and $f$ for females, and the summation is across all time categories at the specified level. Applied to the data used to compile Figure 1, TD equals 150.4 minutes for couple families without children, and 282.6 minutes for couple families with children. In other words, for the time allocations across the five/six categories of time use analysed here to be the same for men and women, either men or women in couple families without children would have to alter their time allocations by two and onehalf hours. In couple families with children this figure is over four and two-thirds hours.

\footnotetext{
${ }^{4}$ There are many other intuitively reasonable patterns evident in these data. Included are the slightly smaller allocations of time to personal care and leisure by both males and females in couple families with children than in couple families without children. See Birch, Le and Miller (2009) for further information.
} 


\section{Figure 1}

Allocation of Time of Males and Females in Couple Families With and Without Children
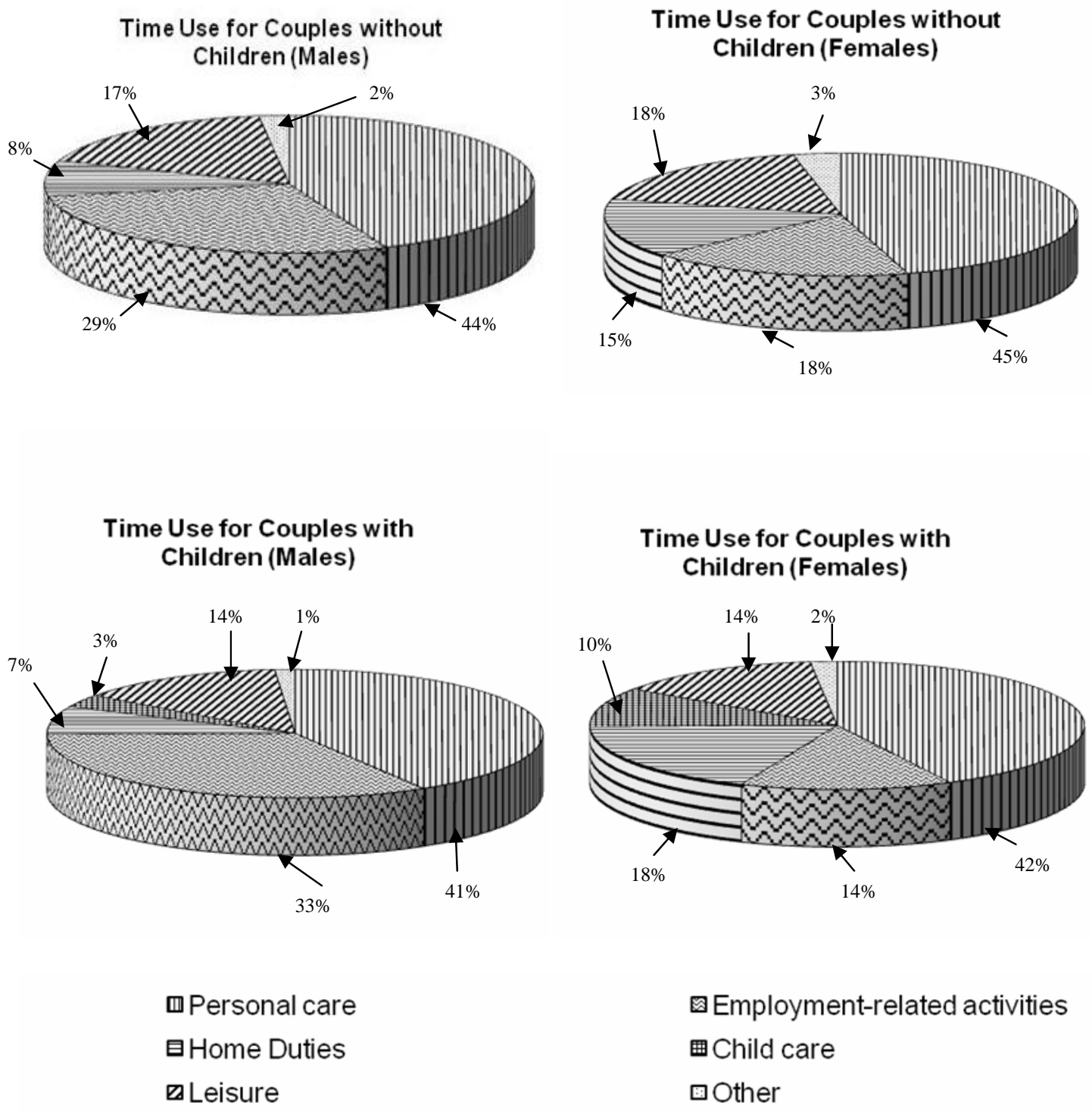

Time Use for Couples with Children (Females)

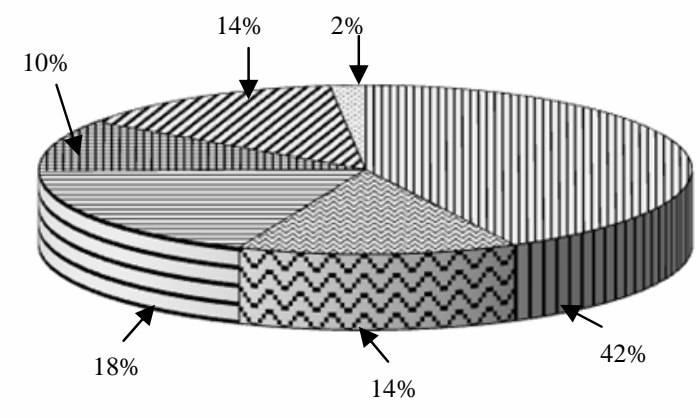

圆Employment-related activities 田Child care ๑Other 
The allocations of time portrayed in Figure 1 are dominated by personal care time, and this might be viewed as non-discretionary. Accordingly, the index of time dissimilarity was also computed after removing personal care time from the calculation. This involves adjusting the time allocations for one partner in a pro-rate fashion to compensate for the minor differences in the time allocated to personal care. Under this scenario, the index of time dissimilarity (TD) was 137 minutes in couple families without children (or 17.4 percent of discretionary time) and 273 minutes in couple families with children (or 33 percent of discretionary time). Clearly, there is considerable gender inequality in the allocation of time in couple families, and children intensify this inequality.

\section{(b) Coordination of Time Use}

The degree of coordination of the activities of partners in couple families can be ascertained through estimating empirical reaction curves. These involve the incorporation of information on one partner's time use in the estimating equation for the other partner. Various approaches may be considered. The data may be analysed using Ordinary Least Squares or Tobit models. Furthermore, a single equation or systems approach could be adopted. A number of specifications of the estimating equation could be used, which differ in the detail on the partner that is included. For example, only information on the partner's time use could be used, or characteristics of the partner (e.g., age, educational attainment) also added to the model (see Birch, Le and Miller, 2009 for discussion). Fortunately, the main findings from this analysis are not sensitive to these issues. Hence, the key findings are conveyed by means of the simplest approach taken: analysis using Ordinary Least Squares and using the 
partner's time allocation to the particular activity as the only variable characterizing the partner.

Table 1 presents results from the statistical analysis of the co-ordination of time use. The estimating equation used to generate these results includes variables for age (eight dummy variables), educational attainment (five dummies), self-reported health status (four dummies), birthplace, length of residence of immigrants in Australia and English proficiency (six variables), area of residence (three variables), home ownership (one variable), and children and the availability of child care (four variables for couple families with children). The partial effects of the variables typically included in time use models are consistent with the literature. Thus, the time allocated to employment-related activities declines after around age 40, and the time allocated to home duties increases around this age. Individuals in poor health allocate less time to employment-related activities and more time to home duties. The better educated have a greater time commitment to the labor market, but educational attainment has only a modest influence on the time allocated to home duties. Children, especially young children, reduce the time allocated to employment-related activities and increase the time allocated to home duties. The effects of children are more pronounced for females than they are for males. 
Table 1

OLS Analysis of Co-ordination of Time Use in Couple Families, Without Children

\begin{tabular}{|c|c|c|c|c|}
\hline \multirow[b]{2}{*}{ Variable } & \multicolumn{2}{|c|}{ Males } & \multicolumn{2}{|c|}{ Females } \\
\hline & $\begin{array}{l}\text { Employment- } \\
\text { related }\end{array}$ & $\begin{array}{l}\text { Home } \\
\text { Duties }\end{array}$ & $\begin{array}{c}\text { Employment } \\
\text {-related }\end{array}$ & $\begin{array}{l}\text { Home } \\
\text { Duties }\end{array}$ \\
\hline Constant & $\begin{array}{l}584.903 \\
(11.25)\end{array}$ & $\begin{array}{l}19.662 \\
(0.79)\end{array}$ & $\begin{array}{c}126.145 \\
(1.90)\end{array}$ & $\begin{array}{c}140.070 \\
(5.17)\end{array}$ \\
\hline \multicolumn{5}{|l|}{ Age (20-24 years) } \\
\hline $25-29$ & $\begin{array}{l}-148.435 \\
(3.14)\end{array}$ & $\begin{array}{l}71.235 \\
(3.44)\end{array}$ & $\begin{array}{l}54.783 \\
(1.13)\end{array}$ & $\begin{array}{l}12.835 \\
(0.61)\end{array}$ \\
\hline $30-34$ & $\begin{array}{c}-64.269 \\
(1.29)\end{array}$ & $\begin{array}{l}26.474 \\
(1.40)\end{array}$ & $\begin{array}{l}2.744 \\
(0.05)\end{array}$ & $\begin{array}{l}63.539 \\
(2.14)\end{array}$ \\
\hline $35-39$ & $\begin{array}{c}-67.628 \\
(1.12)\end{array}$ & $\begin{array}{l}30.306 \\
(1.33)\end{array}$ & $\begin{array}{l}-54.128 \\
(0.84)\end{array}$ & $\begin{array}{c}-19.541 \\
(0.75)\end{array}$ \\
\hline $40-44$ & $\begin{array}{c}-131.119 \\
(2.09)\end{array}$ & $\begin{array}{l}71.390 \\
(2.33)\end{array}$ & $\begin{array}{c}-54.567 \\
(0.85)\end{array}$ & $\begin{array}{l}73.121 \\
(2.31)\end{array}$ \\
\hline $45-49$ & $\begin{array}{l}-178.740 \\
(3.23)\end{array}$ & $\begin{array}{l}62.811 \\
(2.81)\end{array}$ & $\begin{array}{c}-107.854 \\
(2.11)\end{array}$ & $\begin{array}{c}108.718 \\
(4.38)\end{array}$ \\
\hline $50-54$ & $\begin{array}{l}-160.940 \\
(2.60)\end{array}$ & $\begin{array}{c}40.833 \\
(1.64)\end{array}$ & $\begin{array}{l}-110.023 \\
(2.14)\end{array}$ & $\begin{array}{l}78.642 \\
(3.26)\end{array}$ \\
\hline $55-59$ & $\begin{array}{c}-219.320 \\
(4.43)\end{array}$ & $\begin{array}{l}71.479 \\
(3.53)\end{array}$ & $\begin{array}{c}-136.890 \\
(2.87)\end{array}$ & $\begin{array}{c}134.581 \\
(6.17)\end{array}$ \\
\hline $60-64$ & $\begin{array}{c}-303.816 \\
(5.71)\end{array}$ & $\begin{array}{c}110.270 \\
(4.41)\end{array}$ & $\begin{array}{l}-204.219 \\
(3.98)\end{array}$ & $\begin{array}{c}155.968 \\
(5.39)\end{array}$ \\
\hline \multicolumn{5}{|l|}{ Birthplace (Australia) } \\
\hline $\begin{array}{l}\text { Overseas: English- } \\
\text { speaking countries }\end{array}$ & $\begin{array}{l}63.374 \\
(1.75)\end{array}$ & $\begin{array}{l}-12.844 \\
(0.60)\end{array}$ & $\begin{array}{l}-20.900 \\
(0.58)\end{array}$ & $\begin{array}{c}-23.268 \\
(1.04)\end{array}$ \\
\hline $\begin{array}{l}\text { Overseas: non- } \\
\text { Eng.- } \\
\text { speaking countries }\end{array}$ & $\begin{array}{l}45.549 \\
(1.14)\end{array}$ & $\begin{array}{l}0.563 \\
(0.02)\end{array}$ & $\begin{array}{l}-39.104 \\
(0.90)\end{array}$ & $\begin{array}{c}-20.144 \\
(0.84)\end{array}$ \\
\hline \multicolumn{5}{|c|}{ Duration of Residence (20+ years) } \\
\hline $0-10$ years & $\begin{array}{c}38.852 \\
(0.69)\end{array}$ & $\begin{array}{l}-14.230 \\
(0.46)\end{array}$ & $\begin{array}{l}78.548 \\
(1.41)\end{array}$ & $\begin{array}{l}-39.162 \\
(1.30)\end{array}$ \\
\hline $11-20$ years & $\begin{array}{l}0.840 \\
(0.01)\end{array}$ & $\begin{array}{l}21.837 \\
(0.64)\end{array}$ & $\begin{array}{r}89.874 \\
(1.38)\end{array}$ & $\begin{array}{r}53.360 \\
(1.41)\end{array}$ \\
\hline \multicolumn{5}{|c|}{ English Proficiency (speaks only English) } \\
\hline $\begin{array}{l}\text { Speaks English very } \\
\text { well }\end{array}$ & $\begin{array}{c}-189.401 \\
(2.50)\end{array}$ & $\begin{array}{l}71.074 \\
(1.68)\end{array}$ & $\begin{array}{c}153.068 \\
(1.53)\end{array}$ & $\begin{array}{c}-15.552 \\
(0.34)\end{array}$ \\
\hline $\begin{array}{l}\text { Speaks English } \\
\text { well, } \\
\text { not well, not at all } \\
\text { Health (good) }\end{array}$ & $\begin{array}{c}-30.169 \\
(0.36)\end{array}$ & $\begin{array}{l}39.874 \\
(1.03)\end{array}$ & $\begin{array}{l}-42.743 \\
(0.56)\end{array}$ & $\begin{array}{c}24.956 \\
(0.52)\end{array}$ \\
\hline Excellent & $\begin{array}{c}-13.239 \\
(0.41)\end{array}$ & $\begin{array}{c}23.718 \\
(1.27)\end{array}$ & $\begin{array}{l}-4.591 \\
(0.13)\end{array}$ & $\begin{array}{c}40.784 \\
(2.03)\end{array}$ \\
\hline Very good & $\begin{array}{l}30.813 \\
(1.20)\end{array}$ & $\begin{array}{l}-6.308 \\
(0.44)\end{array}$ & $\begin{array}{l}-5.239 \\
(0.20)\end{array}$ & $\begin{array}{l}13.365 \\
(0.91)\end{array}$ \\
\hline
\end{tabular}




\begin{tabular}{|lcccc|} 
Fair & -167.778 & 53.409 & -79.027 & 58.979 \\
Poor & $(4.51)$ & $(2.78)$ & $(2.51)$ & $(2.71)$ \\
& -273.353 & 39.754 & -267.918 & 80.112 \\
Educational Attainment & $(5.30)$ & $(1.71)$ & $(4.26)$ & $(2.06)$ \\
Postghraduate & school graduates) & & & \\
Degree, & 148.975 & -58.852 & 115.862 & -7.536 \\
Graduate Diploma & $(2.28)$ & $(1.73)$ & $(2.21)$ & $(0.23)$ \\
Bachelor's Degree & & & & \\
& 49.146 & -33.216 & 101.545 & -12.029 \\
Advanced Diploma, & $(1.23)$ & $(1.46)$ & $(2.36)$ & $(0.53)$ \\
Diploma & 50.723 & -8.819 & 132.358 & -7.939 \\
Certificate & $(1.26)$ & $(0.39)$ & $(2.70)$ & $(0.29)$ \\
& 50.246 & -20.067 & 57.172 & 5.561 \\
Did not complete & $(1.35)$ & $(0.98)$ & $(1.26)$ & $(0.23)$ \\
high school & 55.717 & -35.873 & 37.993 & 22.491 \\
Area of Residence (middle quintile of areas) & $(1.74)$ & $(0.94)$ & $(1.05)$ \\
Lowest quintile of & -35.840 & -1.358 & 10.664 & -9.336 \\
areas & $(1.16)$ & $(0.09)$ & $(0.32)$ & $(0.48)$ \\
Second lowest & -126.923 & 20.884 & 46.032 & -18.677 \\
quintile of areas & $(3.56)$ & $(1.23)$ & $(1.45)$ & $(0.97)$ \\
Top two quintiles of & -89.786 & 23.109 & 60.142 & -30.585 \\
areas & $(3.06)$ & $(1.44)$ & $(2.16)$ & $(1.87)$ \\
Home owner & 13.062 & 8.304 & 66.336 & -24.292 \\
& $(0.53)$ & $(0.68)$ & $(2.15)$ & $(1.37)$ \\
Partner's allocation of & 0.182 & 0.132 & 0.196 & 0.142 \\
time to same activity & $(4.26)$ & $(2.70)$ & $(4.77)$ & $(2.64)$ \\
\hline$R^{2}$ & 0.327 & 0.142 & 0.256 & 0.212 \\
Sample size & 528 & 528 & 528 & 528 \\
Mean of dependent & & & & \\
variable & 412.25 & 121.52 & 261.80 & 222.35 \\
\hline
\end{tabular}

Note: Absolute value of heteroskedasticity-consistent ' $t$ ' statistics in parentheses. The benchmark group for the categorical variables are given in parentheses against the category name. 
Table 2

OLS Analysis of Co-ordination of Time Use in Couple Families, With Children

\begin{tabular}{|c|c|c|c|c|}
\hline \multirow[b]{2}{*}{ Variable } & \multicolumn{2}{|c|}{ Males } & \multicolumn{2}{|c|}{ Females } \\
\hline & $\begin{array}{c}\text { Employmen } \\
\text { t-related }\end{array}$ & $\begin{array}{l}\text { Home } \\
\text { Duties }\end{array}$ & $\begin{array}{c}\text { Employment } \\
\text {-related }\end{array}$ & $\begin{array}{l}\text { Home } \\
\text { Duties }\end{array}$ \\
\hline Constant & $\begin{array}{l}757.266 \\
(13.77)\end{array}$ & $\begin{array}{c}-22.008 \\
(0.68)\end{array}$ & $\begin{array}{c}290.665 \\
(4.65)\end{array}$ & $\begin{array}{c}237.779 \\
(5.44)\end{array}$ \\
\hline \multicolumn{5}{|l|}{ Age (20-24 years) } \\
\hline $25-29$ & $\begin{array}{c}-93.242 \\
(2.48)\end{array}$ & $\begin{array}{l}44.964 \\
(1.99)\end{array}$ & $\begin{array}{l}-2.976 \\
(0.06)\end{array}$ & $\begin{array}{l}-26.293 \\
(0.72)\end{array}$ \\
\hline $30-34$ & $\begin{array}{c}-174.600 \\
(4.33)\end{array}$ & $\begin{array}{l}88.945 \\
(3.64)\end{array}$ & $\begin{array}{l}12.386 \\
(0.26)\end{array}$ & $\begin{array}{l}6.148 \\
(0.17)\end{array}$ \\
\hline $35-39$ & $\begin{array}{c}-175.863 \\
(4.22)\end{array}$ & $\begin{array}{l}86.577 \\
(3.42)\end{array}$ & $\begin{array}{l}86.167 \\
(1.71)\end{array}$ & $\begin{array}{l}-25.123 \\
(0.69)\end{array}$ \\
\hline $40-44$ & $\begin{array}{c}-219.564 \\
(4.93)\end{array}$ & $\begin{array}{c}101.928 \\
(3.67)\end{array}$ & $\begin{array}{l}60.720 \\
(1.19)\end{array}$ & $\begin{array}{l}-3.617 \\
(0.10)\end{array}$ \\
\hline $45-49$ & $\begin{array}{c}-234.934 \\
(4.90)\end{array}$ & $\begin{array}{c}119.752 \\
(4.25)\end{array}$ & $\begin{array}{c}-18.625 \\
(0.34)\end{array}$ & $\begin{array}{l}24.669 \\
(0.62)\end{array}$ \\
\hline $50-54$ & $\begin{array}{l}-281.106 \\
(5.47)\end{array}$ & $\begin{array}{c}145.231 \\
(4.92)\end{array}$ & $\begin{array}{l}-101.134 \\
(1.69)\end{array}$ & $\begin{array}{l}73.657 \\
(1.65)\end{array}$ \\
\hline $55-59$ & $\begin{array}{c}-338.829 \\
(5.68)\end{array}$ & $\begin{array}{c}135.666 \\
(4.04)\end{array}$ & $\begin{array}{c}-106.235 \\
(1.66)\end{array}$ & $\begin{array}{l}55.903 \\
(1.19)\end{array}$ \\
\hline $60-64$ & $\begin{array}{c}-431.779 \\
(6.53)\end{array}$ & $\begin{array}{c}182.728 \\
(4.73)\end{array}$ & $\begin{array}{c}-70.370 \\
(0.88)\end{array}$ & $\begin{array}{l}71.037 \\
(1.22)\end{array}$ \\
\hline \multicolumn{5}{|c|}{ Birthplace (Australia) } \\
\hline $\begin{array}{l}\text { Overseas: English- } \\
\text { speaking countries }\end{array}$ & $\begin{array}{c}38.441 \\
(1.38)\end{array}$ & $\begin{array}{c}-30.381 \\
(2.29)\end{array}$ & $\begin{array}{l}30.819 \\
(0.98)\end{array}$ & $\begin{array}{l}-13.275 \\
(0.75)\end{array}$ \\
\hline $\begin{array}{l}\text { Overseas: non- } \\
\text { English- } \\
\text { speaking countries }\end{array}$ & $\begin{array}{c}-15.720 \\
(0.55)\end{array}$ & $\begin{array}{l}0.085 \\
(0.01)\end{array}$ & $\begin{array}{l}31.714 \\
(0.96)\end{array}$ & $\begin{array}{l}-5.518 \\
(0.26)\end{array}$ \\
\hline \multicolumn{5}{|c|}{ Duration of Residence (20+ years) } \\
\hline $0-10$ years & $\begin{array}{l}11.818 \\
(0.31)\end{array}$ & $\begin{array}{l}20.327 \\
(0.95)\end{array}$ & $\begin{array}{l}-15.989 \\
(0.41)\end{array}$ & $\begin{array}{l}-15.849 \\
(0.63)\end{array}$ \\
\hline 11-20 years & $\begin{array}{c}-37.018 \\
(0.96)\end{array}$ & $\begin{array}{c}12.119 \\
(0.60)\end{array}$ & $\begin{array}{l}-18.530 \\
(0.48)\end{array}$ & $\begin{array}{l}0.340 \\
(0.01)\end{array}$ \\
\hline \multicolumn{5}{|c|}{ English Proficiency (speaks only English) } \\
\hline $\begin{array}{l}\text { Speaks English } \\
\text { very } \\
\text { well }\end{array}$ & $\begin{array}{l}8.835 \\
(0.19)\end{array}$ & $\begin{array}{l}0.153 \\
(0.01)\end{array}$ & $\begin{array}{r}50.750 \\
(1.12)\end{array}$ & $\begin{array}{c}-39.136 \\
(1.20)\end{array}$ \\
\hline $\begin{array}{l}\text { Speaks English } \\
\text { well, } \\
\text { not well, not at all } \\
\text { Health (good) }\end{array}$ & $\begin{array}{c}-73.825 \\
(1.72)\end{array}$ & $\begin{array}{l}17.669 \\
(0.77)\end{array}$ & $\begin{array}{c}-103.250 \\
(2.84)\end{array}$ & $\begin{array}{l}41.015 \\
(1.60)\end{array}$ \\
\hline Excellent & $\begin{array}{c}-12.566 \\
(0.58)\end{array}$ & $\begin{array}{c}-17.309 \\
(1.74)\end{array}$ & $\begin{array}{l}52.366 \\
(2.43)\end{array}$ & $\begin{array}{c}-31.050 \\
(2.29)\end{array}$ \\
\hline Very good & $\begin{array}{l}16.486 \\
(0.97)\end{array}$ & $\begin{array}{l}-8.759 \\
(0.95)\end{array}$ & $\begin{array}{c}30.446 \\
(1.74)\end{array}$ & $\begin{array}{l}1.079 \\
(0.10)\end{array}$ \\
\hline
\end{tabular}




\begin{tabular}{|c|c|c|c|c|}
\hline Fair & $\begin{array}{c}-70.456 \\
(1.74)\end{array}$ & $\begin{array}{l}3.976 \\
(0.19)\end{array}$ & $\begin{array}{c}-32.822 \\
(1.02)\end{array}$ & $\begin{array}{l}32.647 \\
(1.34)\end{array}$ \\
\hline Poor & $\begin{array}{c}-321.698 \\
(5.77)\end{array}$ & $\begin{array}{l}39.327 \\
(1.06)\end{array}$ & $\begin{array}{l}87.433 \\
(1.33)\end{array}$ & $\begin{array}{l}-56.745 \\
(1.38)\end{array}$ \\
\hline \multicolumn{5}{|c|}{ Educational Attainment (high school graduates) } \\
\hline $\begin{array}{l}\text { Postgraduate } \\
\text { Degree, } \\
\text { Graduate Diploma }\end{array}$ & $\begin{array}{l}8.870 \\
(0.23)\end{array}$ & $\begin{array}{c}-17.880 \\
(0.89)\end{array}$ & $\begin{array}{l}79.755 \\
(2.62)\end{array}$ & $\begin{array}{l}-78.201 \\
(4.12)\end{array}$ \\
\hline Bachelor's Degree & $\begin{array}{l}34.298 \\
(1.27)\end{array}$ & $\begin{array}{c}-18.150 \\
(1.29)\end{array}$ & $\begin{array}{l}46.074 \\
(1.90)\end{array}$ & $\begin{array}{l}-52.503 \\
(3.43)\end{array}$ \\
\hline $\begin{array}{l}\text { Advanced } \\
\text { Diploma, } \\
\text { Diploma }\end{array}$ & $\begin{array}{l}22.415 \\
(0.74)\end{array}$ & $\begin{array}{c}-13.020 \\
(0.86)\end{array}$ & $\begin{array}{l}20.326 \\
(0.75)\end{array}$ & $\begin{array}{c}-34.213 \\
(1.98)\end{array}$ \\
\hline Certificate & $\begin{array}{l}20.074 \\
(0.86)\end{array}$ & $\begin{array}{l}-16.658 \\
(1.30)\end{array}$ & $\begin{array}{l}41.978 \\
(1.57)\end{array}$ & $\begin{array}{l}-40.567 \\
(2.32)\end{array}$ \\
\hline $\begin{array}{l}\text { Did not complete } \\
\text { high } \\
\text { school }\end{array}$ & $\begin{array}{l}-9.797 \\
(0.36)\end{array}$ & $\begin{array}{l}-5.823 \\
(0.40)\end{array}$ & $\begin{array}{c}-60.176 \\
(2.79)\end{array}$ & $\begin{array}{l}5.680 \\
(0.39)\end{array}$ \\
\hline \multicolumn{5}{|c|}{ Area of Residence (middle quintile of areas) } \\
\hline $\begin{array}{l}\text { Lowest quintile of } \\
\text { areas }\end{array}$ & $\begin{array}{c}-74.424 \\
(2.78)\end{array}$ & $\begin{array}{c}32.423 \\
(2.25)\end{array}$ & $\begin{array}{l}3.551 \\
(0.15)\end{array}$ & $\begin{array}{l}-10.097 \\
(0.62)\end{array}$ \\
\hline $\begin{array}{l}\text { Second lowest } \\
\text { quintile of areas }\end{array}$ & $\begin{array}{c}-49.986 \\
(1.94)\end{array}$ & $\begin{array}{l}22.744 \\
(1.63)\end{array}$ & $\begin{array}{c}-18.386 \\
(0.83)\end{array}$ & $\begin{array}{c}-11.336 \\
(0.76)\end{array}$ \\
\hline $\begin{array}{l}\text { Top two quintiles } \\
\text { of } \\
\text { areas }\end{array}$ & $\begin{array}{l}3.601 \\
(0.20)\end{array}$ & $\begin{array}{l}-7.809 \\
(0.81)\end{array}$ & $\begin{array}{c}35.985 \\
(1.81)\end{array}$ & $\begin{array}{c}-17.006 \\
(1.36)\end{array}$ \\
\hline Home owner & $\begin{array}{l}21.483 \\
(0.96)\end{array}$ & $\begin{array}{l}16.458 \\
(1.54)\end{array}$ & $\begin{array}{c}-15.720 \\
(0.72)\end{array}$ & $\begin{array}{c}23.145 \\
(1.81)\end{array}$ \\
\hline \multicolumn{5}{|c|}{ Age of Youngest Child (25+ years) } \\
\hline $0-4$ & $\begin{array}{c}-99.916 \\
(2.56)\end{array}$ & $\begin{array}{l}40.002 \\
(2.01)\end{array}$ & $\begin{array}{c}-271.887 \\
(6.88)\end{array}$ & $\begin{array}{l}83.773 \\
(3.09)\end{array}$ \\
\hline $5-12$ & $\begin{array}{c}-16.550 \\
(0.48)\end{array}$ & $\begin{array}{l}-0.326 \\
(0.02)\end{array}$ & $\begin{array}{c}-137.641 \\
(3.82)\end{array}$ & $\begin{array}{l}60.922 \\
(2.44)\end{array}$ \\
\hline $13-25$ & $\begin{array}{c}-34.317 \\
(1.06)\end{array}$ & $\begin{array}{l}39.106 \\
(2.21)\end{array}$ & $\begin{array}{l}22.620 \\
(0.74)\end{array}$ & $\begin{array}{c}26.188 \\
(1.30)\end{array}$ \\
\hline Child care available & $\begin{array}{l}14.172 \\
(0.76)\end{array}$ & $\begin{array}{l}5.789 \\
(0.66)\end{array}$ & $\begin{array}{l}49.664 \\
(2.74)\end{array}$ & $\begin{array}{l}-24.496 \\
(2.03)\end{array}$ \\
\hline $\begin{array}{l}\text { Partner's allocation } \\
\text { of time to same } \\
\text { activity }\end{array}$ & $\begin{array}{l}-0.054 \\
(1.61)\end{array}$ & $\begin{array}{l}-0.044 \\
(1.60)\end{array}$ & $\begin{array}{l}-0.041 \\
(1.36)\end{array}$ & $\begin{array}{l}-0.040 \\
(1.04)\end{array}$ \\
\hline$R^{2}$ & 0.138 & 0.089 & 0.196 & 0.091 \\
\hline Sample size & 1,012 & 1,102 & 1,012 & 1,012 \\
\hline $\begin{array}{l}\text { Mean of dependent } \\
\text { variable }\end{array}$ & 481.62 & 102.08 & 199.01 & 263.84 \\
\hline
\end{tabular}

Note: Absolute value of heteroskedasticity-consistent ' $t$ ' statistics in parentheses. The benchmark group for the categorical variables are given in parentheses against the category name. 
The links between the time allocations of partners from these results are presented in graphical form in Figure 2. This figure contains reaction curves for partners in couple families for employment-related activities in the top panel, and for home duties in the bottom panel. The figures on the left-hand side are for couple families without children, while those on the right-hand side are for couple families with children.

Partners' activities in couple families without children are characterised by complementarity: the male (female) partner's allocation of time to a particular activity is significantly and positively related to the time use of his wife (her husband). Each additional hour allocated to the labor market by the husband (wife) is associated with 20 (18) additional minutes allocated to labor market activities by the wife (husband). Similarly, each additional hour of time allocated to home duties by the husband (wife) is associated with 14 (13) extra minutes of home duty time on the part of the wife (husband). This complementarity can co-exist with specialization because of shifts in the intercept terms of the reaction curves. However, the complementarity moderates the degree of specialization that would otherwise be observed in these data.

The time allocations of partners in couple families with children differ from those for partners in couple families without children in two respects. First, the statistically significant and positive relationships between the partners' time allocations are replaced by negative, though statistically insignificant relationships. Second, there are further shifts in the intercepts of the reaction curves to reflect the more intense specialization that takes place in the presence of children. Consequently, males in couple families with children allocate around 482 minutes to employment-related activities, compared to 412 minutes in couple families without 
children. Females in couple families with children allocate 264 minutes to home duties compared to 222 minutes in couple families without children.

Figure 2

\section{Coordination of Employment-related Activities and Home Duties of Males and Females in Couple Families With and Without Children}
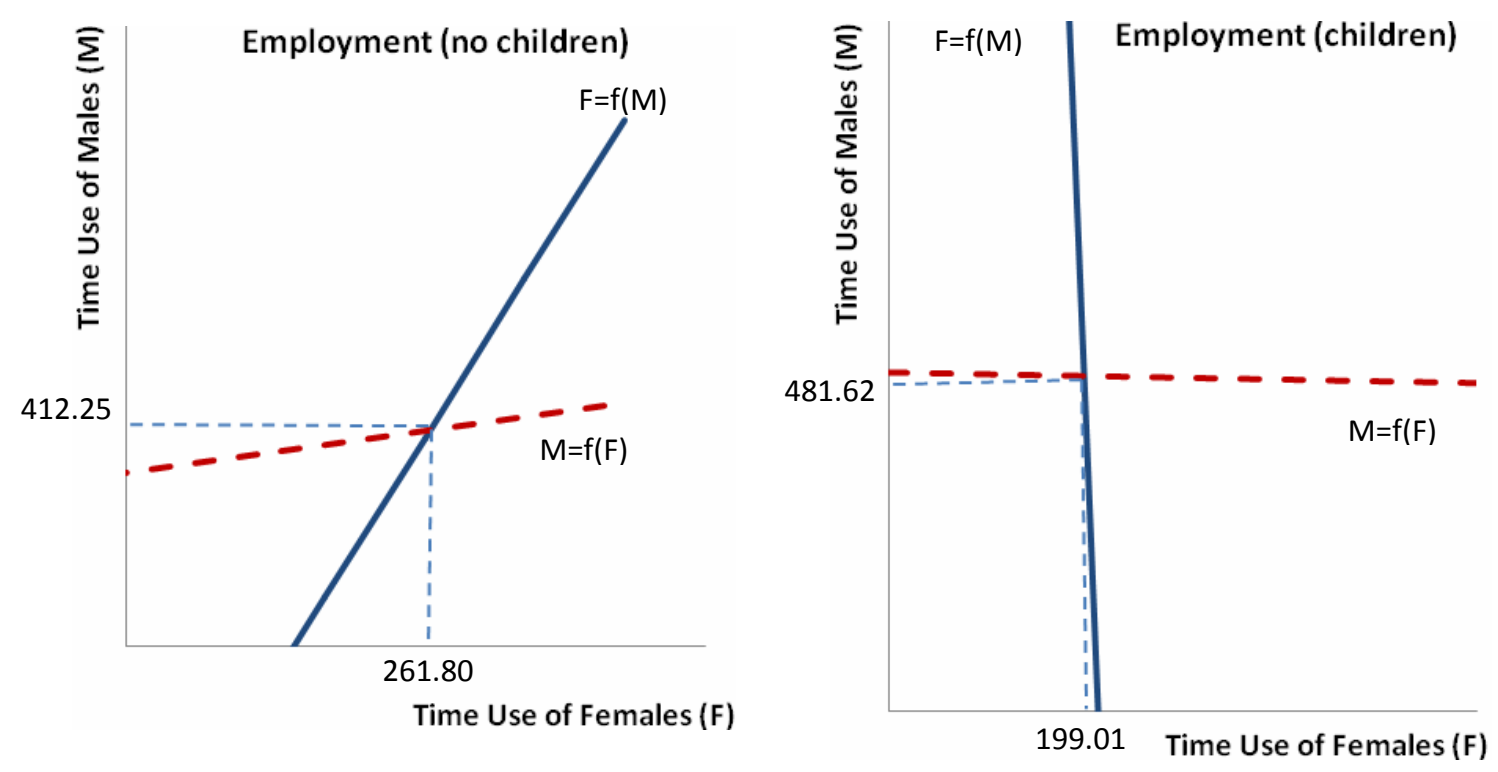

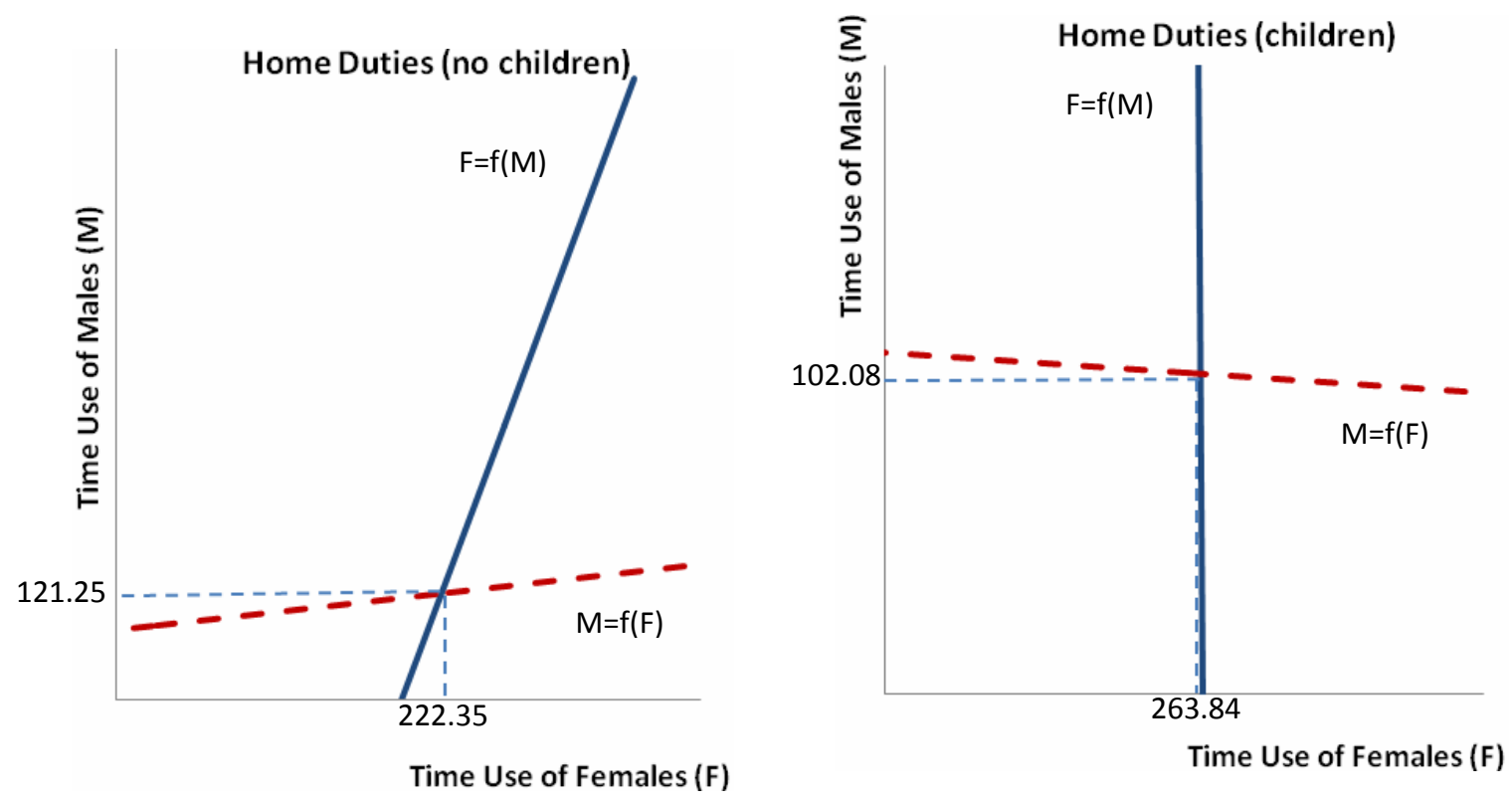

This change in the way the time allocations of partners in couple families are linked in the presence of children carries over to estimations where the time use equations for partners in couple families are estimated jointly. Under this approach, in couple families with children, there is a statistically significant, positive correlation between the error terms on the time use equations for both males and females, both for the analysis of employment-related activities and home duties. In other words, the unobservables associated with a relatively high allocation of time to employmentrelated activities by one partner in a couple family without children are positively correlated with the unobservables associated with a relatively high allocation of time to these activities by the other partner. In the case of couple families with children, however, the error terms in the equations for both these categories of time use are negatively correlated, thought the correlation is not statistically significant. Children, 
therefore, appear to remove the scope for parents to undertaken similar activities: they need to specialize.

Thus, in couple families without children, specialization in specific time uses coexists with coordination or complementarity of time use, and this coordination serves to moderate the degree of specialization. In couple families with children, this complementarity of time use is absent, which intensifies the degree of specialization.

\section{CONCLUSION}

In order for the time allocations of partners in couple families without children to be congruent, around two and one-half hours would need to be reallocated across major time uses by one of the partners. In couple families with children, the comparable figure is four and two-thirds hours. Children clearly intensify the specialization in time use activities within the family. If personal care-which is largely non-discretionary-is removed from the calculations, around 17 percent of discretionary time would have to be reallocated across activities by partners in couple families without children for there to be congruency of time use of the partners. In couple families with children this figure is as high as 33 percent.

The way in which this more intense specialisation comes about in the presence of children appears to be through the elimination of the complementarity of time uses of partners in couple families. Thus, in couple families where there are no children present, partners' time uses are coordinated in the sense that additional time set aside for a particular activity by one partner is associated with additional time for that activity by the other partner. In couple families with children, however, the time uses of partners are not related in this sense. Nor are the unobservables that are associated with relatively high time use by one partner linked to the unobservables that are 
associated with relatively high time use by the other partner. Children thus intensify the specialization in time use by moderating the scope for partners to pursue similar, complementary, time allocations. 


\section{REFERENCES}

Becker, G. (1991). A Treatise on the Family, Harvard University Press, Cambridge, United States.

Birch, E. R., Le, A. T. and Miller, P. W., (2009). Household Divisions of Labour: Teamwork, Gender and Time, Palgrave Macmillan Studies in Family and Intimate Life, Basingstoke, United Kingdom.

Duncan, O. D., and Duncan, B. (1955), 'A methodological analysis of segregation indexes’, American Sociological Review, Vol. 20 (2), pp. 210-217.

Manser, M., and Brown, M. (1980). 'Marriage and Household Decision Making: A Bargaining Analysis’, International Economic Review Vol. 21, pp.31-34. 